\title{
An Experimental Comparison of the Effectiveness of Control Flow Based Testing Approaches on Seeded Faults
}

\author{
Atul Gupta and Pankaj Jalote \\ Dept. of Computer Science and Engineering, \\ Indian Institute of Technology Kanpur 208 016, India \\ \{atulkg, jalote\}@cse.iitk.ac.in
}

\begin{abstract}
In this paper, we describe the results of an experiment comparing the effectiveness of three structural coverage-testing methods, namely, block coverage, branch coverage and predicate coverage criteria on seeded faults. The implications of our work is two-fold: one, we describe a controlled simulation comparing the effectiveness of these criteria and two, we demonstrate a novel approach to generate minimal test suites for these coverage criteria so as to be able to predict relative performance of the three coverage-adequate test suites. Using a byte code coverage analyzer, five java programs of different sizes were tested. Faults were seeded in all five programs using a set of applicable mutation operators. Twenty-five different minimal JUnit test suites were then constructed for each coverage criteria-program pair and executed on program's mutants to compare the effectiveness. Results suggest that generally, branch coverage criterion performed consistently and was found to be most viable option for structural testing. However, in presence of composite conditions, predicate testing does better and its effectiveness increases with increase in the cardinality of the composite conditions.
\end{abstract}

Keywords: Block coverage, Branch coverage, Predicate coverage, Software testing, Test case generation, Experiment, Mutation Operator, Statistical analysis.

\section{Introduction}

Last two decades have seen rapid growth of research in software testing, design and analysis of experiments. Experimentation in Software Engineering supports the advancement of the field through an iterative learning process and software testing is no exception [4,5]. In the mid 70's Goodenough and Gerhart 13] put forward perhaps the most important question in software testing: What is a test criterion for an adequate testing? Since then testing criteria has been a major research focus. A great number of criteria have been proposed and investigated 15. E. J. Weyuker highlighted that measuring the effectiveness of testing is generally not possible, but that comparison is 23,24 . Comparing various criteria systematically requires them to be classified and one well accepted classification of the test adequacy criteria is by underlying testing approach where there are 
three basic approaches to software testing: structural testing, fault based testing and error based testing.

Two main groups of program based structural testing are control flow testing and data flow testing. The structural testing approaches are based on the flow graph model of program structure. A basic requirement of many coverage criteria is that all nodes and edges in the program are covered by test executions. But all nodes or edges executions cannot always be achieved because of possible existence of infeasible statements or edges. The problem to identify whether a piece of code is unreachable is undecidable [11,12. Because of this unreachability we cannot always attain $100 \%$ coverage of the program in terms of statements and branches and criteria like $90 \%$ coverage are used in practice.

A number of comparisons and evaluations of testing adequacy criteria using statistical methods have been studied and investigated by researchers [6, 9, 19, Ntafos 19 compared branch coverage, random testing and required pair coverage with 14 small programs. Test cases for each program were selected from a large set of random test cases and faults are inserted artificially in the programs (mutants). The percentages of mutants killed by the test suite were considered as the fault detection ability. The experiment was identified to have invalidating factors which can influence the findings, by Hamlet [14].

To encounter potentially invalidating factors, Basily and Selby [6] used fractional factorial design methodology of replicated statistical experiments. In their comprehensive experiment, they compared code reading, functional testing and statement coverage method in three distinct phases involving 74 subjects (testers) of different background with four programs. The programs contained natural as well as artificial faults. Authors reported about fault detecting abilities of these testing strategies and efforts with respect to various classes of faults and subjects were compared. In a replicated study of effectiveness of the three testing methods, M. Wood et al. 25] have pointed out that overall effectiveness of these testing methods is similar in terms of observing failures and finding faults but their relative effectiveness depends on the nature of the program and it's faults.

E. J. Weyuker, et al 24] demonstrated that in comparing testing methods the probabilistic comparison, i.e. comparing their effectiveness based on some randomly drawn test suites satisfying desired coverage criteria, has better diagnostics than analytical comparison of testing methods. Frankl and Weiss [9] used this approach to address potential invalidating factors associated with test data. They compared branch coverage and all-uses data flow coverage criteria using 9 small programs. Instead of using one adequate test suite for each criterion, they generated a large number of adequate test suites and used proportion of the test suites that detect errors as an estimate of the probability of detecting errors.

It has been pointed out that branch coverage criterion is stronger than statement coverage because if all edges in a flow graph are covered, all nodes are necessarily covered. Therefore a test suite that satisfies the branch coverage must also satisfy statement coverage. That is branch coverage subsumes statement coverage. Interestingly, Frankl and Weyuker [11] proved that the fact that 
criterion C1 SUBSUMES C2 does not always guarantee that C1 is better at detecting faults.

Though a number of studies have been performed to compare and evaluate different testing strategies, various control flow structural testing approaches like block, branch, predicate coverage, etc. have not been compared experimentally 18. As theoretically branch coverage should do better than statement coverage, one might choose this to do a more through testing. However, statement coverage analyzers are generally easier to build and results are easier to interpret. Hence, if in practice the above two are similar, then one may choose to use statement coverage only. Similarly, predicate coverage seems to require a higher number of test cases and hence more effort but at the same time may have better fault detecting abilities. It would be useful to understand the effectiveness of these different structural coverage criteria and the kinds of faults for which they are more effective. Studying this effectiveness is the main goal of our experiment. The experiment we have conducted also aims to provide some understanding about conditions under which one criterion is more effective than the other.

In our experiment, we have used five Java programs. For each program we created many mutants for detecting the testing effectiveness. To test a program, we created twenty-five different minimal test suites for each coverage criterion using an algorithm to find the minimum number of test cases from a test pool to satisfy that criterion. Performance data like number of mutants killed by the test suites for the three coverage criteria and coverage information for each test suite were obtained. Our experiment confirms that testing effectiveness of the criteria are affected by the program structure, however, in most of the cases, branch coverage performed consistently and needed more effort than block coverage but considerably less effort for predicate coverage. Our results also suggest that for testing a given program, branch coverage perform better than block coverage, irrespective of the program structure and confirmed the same at statistically significance $\alpha=0.01$. When compared to predicate coverage, the later does better in programs with composite conditions in them, with statistical significance of 0.05 . In other cases, branch and predicate coverage have shown similar effectiveness.

The rest of the paper is organized as follows. Its underlying experimental approach is presented in Section 2, Data collection and analysis methods used for comparison are explained in Section 3. Results of the experiments are presented in Section 4. Threats to validity of our results are highlighted in Section [5] and conclusions are given in Section 6 .

\section{Experimental Setup}

Experimentation is an important method in software engineering for understanding the processes and products. To help the researchers, guidelines [4, 5, 7, 21] are laid down so as to design and conduct controlled experiments whose results can be relied upon. In our study, we have taken great care to follow the guidelines and controlling the variability that can be caused by known and unknown factors. 


\subsection{Goals}

The goals of our experiment are to answer the following questions.

- Which coverage criterion has more fault detection ability?

- Which coverage criterion needs more testing effort?

- Are there any specific types of bugs which results in different effectiveness?

- Is there any co-relation between the parameters of the program and testing approaches.

- How one type of coverage is related with others?

- How to choose a suitable criterion for a given program?

\subsection{Some Definitions}

Test Case - A set of inputs, execution preconditions and expected outcomes developed for a particular objective.

Test Suite - A collection of one or more test cases for the software under test normally having some completeness criterion.

Minimal Test Suite - A test suite with minimum number of test cases to satisfy a given criterion.

Test-Pool - A collection of a large number of test cases which can be used for generation of test suites by selecting a subset of test cases from the pool.

Mutation Operator - A handle to seed faults in a program in some specific context.

Mutant - A faulty version of a correct program containing exactly one fault. It is obtained by seeding a fault by applying a mutation operator at some place in that program.

Block Coverage - A block is a set of sequential statements not having any inbetween flow of control, both inward and outward. Complete block coverage requires that every such block in the program be exercised at least once.

Branch Coverage - Complete branch coverage requires that every branch be exercised at least once in both the TRUE and FALSE directions.

Predicate Coverage (or Multi-Condition Coverage) - It requires that each of the logical expressions in a logical expression must evaluate to TRUE in some test, and to FALSE in some other test.

\subsection{Criteria for Comparison}

To answer the questions above we need suitable metrics. To measure the fault detection effectiveness, we use the percentage of faults that are revealed. By keeping it as percentage, it helps generalize across programs. The criteria used to measure fault detection ability $F D E_{T}$ of a test suite $\mathrm{T}$ is

$$
F D E_{T}=\frac{\text { number of faults that are revealed }}{\text { total number of faults present in the program }} \times 100 \%
$$


The criteia used to measure testing effort $T E_{T}$ of a test suite $\mathrm{T}$ is

$T E_{T}=$ Number of test cases in the test suite $\mathrm{T}$ needed to satisfy a given coverage criterion

To understand the connection of fault detecting effectiveness with efforts required in testing, we have used a metric called performance index $P I_{T}$ of a test suite $\mathrm{T}$, which is obtained as

$$
P I_{T}=\frac{\text { Number of faults revealed by test suite } \mathrm{T}}{\text { Size of the test suite } \mathrm{T}} .
$$

\subsection{Instruments Used}

Test Programs. Results obtained from small programs are difficult to generalize [19]. In this study, we have considered moderately sized programs ranging 300 to 1500 lines of code. The programs are taken from diversified domains with general-purpose use to increase applicability of our results. The first program is PostalCodes, which aims at recognizing postal codes of a few countries. The second program, HotelManagement, is a simple command line hotel management application program written by the first author. CruiseControl [27] is the third program that is widely used for state based testing experiments. JavaVector is the Vector class in the Java Collection framework. Our fifth program is a game Monopoly [28], which is quite object-oriented in nature. Programs were written in Java, though we believe that the results of this experiment can be applicable to programs written in other object-oriented languages as well. Important statistics of these programs are given in table 1 .

Faults and Mutants. Selecting test programs is very critical to the study and equally important issue is the bugs they contain. The number of bugs naturally committed by the developers typically much less than what is needed in such an experiment. The same is also true about the types of bugs. If bugs are introduced manually without great care, then it risks invalidating experimental findings. Most recently, Andrews et al. 1] demonstrated that mutation faults can in fact be representative of real faults, and advocated the use of mutation for effective testing experiments, although more studies are needed to generalize their conclusions.

Table 1. Subject Programs statistics

\begin{tabular}{|l|l|l|l|l|l|l|l|l|}
\hline $\begin{array}{l}\text { Sl. } \\
\text { No. }\end{array}$ & $\begin{array}{l}\text { Program } \\
\text { Name }\end{array}$ & $\begin{array}{l}\text { LOC } \\
\text { Faults/ } \\
\text { Mutants }\end{array}$ & $\begin{array}{l}\text { \# of } \\
\text { Classes/faults } \\
\text { seeded in }\end{array}$ & $\begin{array}{l}\text { \# of } \\
\text { Blocks }\end{array}$ & $\begin{array}{l}\text { \# of } \\
\text { Branches }\end{array}$ & $\begin{array}{l}\text { \# of } \\
\text { Predi- } \\
\text { cates }\end{array}$ & $\begin{array}{l}\text { Test- } \\
\text { Pool } \\
\text { size }\end{array}$ \\
\hline \hline 1 & HotelManagement & 450 & 56 & $6 / 4$ & 118 & 136 & 40 & 55 \\
2 & PostalCodes & 340 & 93 & $6 / 4$ & 107 & 134 & 55 & 105 \\
3 & CruiseControl & 540 & 41 & $6 / 4$ & 100 & 105 & 33 & 72 \\
4 & JavaVector & 300 & 72 & $1 / 1$ & 142 & 161 & 39 & 70 \\
5 & Monopoly & 1600 & 56 & $34 / 8$ & 270 & 294 & 55 & 84 \\
\hline
\end{tabular}


A number of studies have used a controlled approach of inserting bugs in programs [2, 3], based on some set of mutation operators as proposed by [16]. We followed a similar approach in which a set of mutants of a program was obtained by seeding faults in the program using a set of applicable mutation operators. Bugs were introduced in the model part of the code and user interface part (GUI) and setter-getter methods were kept bug-free to avoid unwanted interaction between buggy code with testing code.

The mutation operators used and the kinds of faults inserted by them, in this study were:

- Literal Change Operator (LCO) - changing increment to decrement or vice versa, incorrect or missing initialization or increment, incorrect or missing state assignment.

- Language Operator Replacement (LOR) - replacing a relational or logical operator by another.

- Control Flow Disruption (CFD) - missing or incorrectly placed block markers, break, continue or return.

- Method Name Replacement (MNR) - replacing a method with other method of similar definition but different behavior.

- Statement Swap Operator (SSO) - swapping two statement in the same scope.

- Argument Order Interchange (AOI) - interchanging arguments of the same type of a method in the definition or in method-call.

- Variable Replacement Operator (VRO) - replacing a variable with other of similar type.

- Missing Condition Operator (MCO) - Missing out a condition in a composite conditional statement.

- Null Reference Operator (NRO) - Causing a null reference.

Test-Pool. It is a known fact that different test suites for the same criteria may lead to different kind of conclusions [9,23]. To counter this, we have used a definitive procedure of generating test suites from a large test-pool, which were guided by the coverage information as to reach desired coverage early. The testpool was constructed by writing JUnit [26] tests for each non-trivial method of the model classes in the program. It contained enough tests as to generate coverage-adequate test suites for the code under test. It was also ensured that the pool contains at least one test case for each mutant of the program to kill and hence cover the structure of the mutants under study. Constructing all the three coverage adequate test suites from the same test-pool, we ensured a fair comparison to be possible among the three coverage criteria.

Coverage Adequate Minimal Test Suites. Twenty-five test suites for each coverage criterion for each program were generated to statistically analyze the behavior of the three coverage criteria under study. For each program, test casewise coverage information for all coverage criteria was obtained by executing the test-pool on the correct version of the program. Coverage adequate test suites 
then can be generated by simply picking random test cases from the test-pool but this approach will not produce minimal test suites for obvious reasons such as redundencies in elements covered by different test cases in a test suite. One of the motivation of this work is to evaluate the performance of coverage adequate test suites which is possible if we use minimal test suites. Coverage-adequate minimal test suites for a program were constructed using following steps. For constructing each minimal test suite $\mathrm{T}$ :

1. Select first test case randomly from the test-pool, remove it from the pool and add it to $\mathrm{T}$.

2. Update the coverage information for all other remaining test cases in the test-pool so as to incorporate yet-to-cover elements in the test suite $\mathrm{T}$ being so constructed.

3. Select a test case which provides maximum yet-to-cover coverage $(>0)$ and add it to $\mathrm{T}$.

4. Repeat step 2 and 3 until no more test cases can be added to T.

Monitoring Coverage and Testing. To obtain various coverage information, a locally developed tool named JavaCoverage was used [22. This tool provides coverage information for each test case visually using different colors as to represent uncovered program elements, and therefore, effectively guides testers to design and execute further tests to meet testing objectives. The tool does program analysis at byte code level and use byte code instrumentation to obtain various coverage information from test case executions. The coverage information for each test case is recorded in a MySql database.

Each program was tested by executing all coverage-adequate test suites on all its mutants. The information regarding killed-mutants was recorded.

\section{Data Collection and Analysis}

Twenty-five test suites for each criterion were exercised on each of the five programs' mutants. For each program, the following data were recorded and analyzed:

- Number and type of mutants generated and killed by the coverage test suites

- All-coverage information of coverage test suites

- Number of blocks, branches, predicates, program size, test suite size.

Observations obtained for all five programs were separately analyzed to determine the effectiveness of the three coverage criteria. Subsequently, results were also compared across the programs.

\subsection{Program-Wise Analysis}

For each program, we graphically demonstrate (i) faults seeded as per the mutation operator used, (ii) average $F D E_{T}$ of the three coverage criteria, (iii) average coverage achived by the three coverage adequate test suites and (iv) average $P I_{T}$ of these coverage adequate test suites in Figure 1 at the end of this paper. We briefly discuss the program-wise results here. 
Program-1: PostalCodes. The results showed that fault detecting effectiveness $F D E_{T}$ of predicate coverage test suites were better than branch and block coverage test suites but required more testing effort $T E_{T}$. It has shown much better results in the case of missing condition bugs (seeded using MCO operator). Block coverage based test suites has shown better performance index $P I_{T}$ of the test suites then the other two but shown larger variability in the results. They showed poor performance in case of control flow bugs seeded by CFD and MCO operators. Branch coverage test suites identified more bugs than block test suites and their $P I_{T}$ was more stable.

Remarks: This Program has some algorithmic methods with many composite conditional statements. Hence predicate and branch coverage did perform well where as block coverage performed rather poorly and inconsistently.

Program-2: HotelManagement. The results showed that in this case also predicate coverage based test suites show better $F D E_{T}$ but more $T E_{T}$ than the other two. The box plot for $P I_{T}$ shows that overall branch test suites performed better and block test suites performed poorly.

Remarks: This program also has some methods with composite conditions but of less cardinality than Postal Codes. We observed similar results here to that of Postal Code.

Program-3: CruiseControl. The $F D E_{T}$ of the predicate coverage criterion were found to be similar to branch coverage with the fact that branch test suites were also good at MCO bugs. Block tests showed better $P I_{T}$ than other two but with highest variability. Once again branch test suites were consistent and proved to be better than predicate tests. Block test suites $P I_{T}$ was significantly better but having higher variance than the two other.

Remarks: This program consists of mostly small methods with states and transitions. The conditionals are simple-if statements and therefore branch test suite is somewhat larger than block test suites and consequently having considerably less $P I_{T}$. MCO bugs clearly dominate when there are conditionals of high cardinality, which wasn't the case here, and branch test suites did perform well in these conditions.

Program-4: Java Vector. The $F D E_{T}$ of the three coverage criteria was found to be quite different than the above three programs. Branch test performed best followed by block followed by predicate tests. Their $T E_{T}$ requirements were in opposite order, i.e. branch coverage test suites size was more followed by block test suites followed by predicate test suites. Three test suites demonstrated similar $P I_{T}$ with predicate tests showing greatest variability.

Remarks: The reason for the diversity in results is due to the fact that there are many methods in JavaVector that do not contain any conditionals and hence predicate coverage test suites were unlikely to cover those. Also most of the conditionals were simple and hence branch and block test suites have shown similar behavior. 
Program-5: Monopoly. This is a nicely designed object-oriented program as it has inheritance hierarchies with small method sizes and other object-oriented features. Many classes in the model part of the code are small and the methods of the controller classes are well re-factored [8] to perform single functions. Intramethod structural complexity is quite low but inter-method interaction is high. The results of this program showed similar trends as previously obtained but with lesser variability. The three coverage criteria seem to have similar $F D E_{T}, T E_{T}$ and $P I_{T}$ measures. Control flow bugs like MCO and CFD bugs were remained as the cause of difference in $F D E_{T}$ of the three criteria.

Remarks: The results are interesting in the sense that the three coverage criteria show quite similarity in the results, importantly, size of the test suites and corresponding $P I_{T}$. We found comparatively fewer places to seed the kinds of faults, under study, to be inserted. This explains that object-oriented programs are to be tested with a focus on inter-method interactions rather than intra-method control flow.

\subsection{Program Analysis at Method Scope}

To obtain statistical evidences for coverage criteria effectiveness, we have investigated some important methods of the classes of these five programs. We collected various statistics of these methods like size, number of blocks, branches and predicates, number of faults inserted, faults identified by the coverage test suites, etc. The reason for doing analysis at method level is that it provides more data points, thereby allowing better statistical analysis of the results obtained. Since the control structure is typically represented at method level, analysis at method level is the right level of granularity for coverage analysis. Please refer to table 2 for the statistical analysis results presented in this subsection where $\mu$ denotes mean of effectiveness parameter in consideration i.e. $F D E_{T}$ over twenty five test suites and subscripts $\mathrm{Bl}, \mathrm{Br}$, and Pr represent block, branch and predicate coverage criteria, respectively.

Our results show that $F D E_{T}$ of branch coverage outperformed block coverage with statistical significance of $\alpha=0.01$ (row 1 table 2). When branch coverage is compared with predicate coverage, from the experiment data, we found that there is not enough evidence to reject the null hypothesis and both coverage criteria have shown similar $F D E_{T}$ (row 2 table 2). However, when we drill down further, we found that predicate coverage criterion performs better then branch criterion in methods which contains composite conditional statements. This observation was confirmed statistically at a significance level of $\alpha=0.05$ (row 3 table 2).

Table 2. Method-level Analysis Results

\begin{tabular}{|l|l|l|l|l|l|l|l|}
\hline $\begin{array}{l}\text { Sl. } \\
\text { No. }\end{array}$ & $\begin{array}{l}\text { Effectiveness } \\
\text { Parameter }\end{array}$ & $\begin{array}{l}\text { Null } \\
\text { Hypothesis }\end{array}$ & $\begin{array}{l}\text { Alternate } \\
\text { Hypothesis }\end{array}$ & t-value & t-critical & p-value & Result \\
\hline \hline 1 & $F D E_{T}$ & $\mu_{B r}=\mu_{B l}$ & $\mu_{B r}>\mu_{B l}$ & 3.612 & 2.079 & 0.001 & $\mu_{B r}>\mu_{B l}$ \\
2 & $F D E_{T}$ & $\mu_{P r}=\mu_{B r}$ & $\mu_{P r}>\mu_{B r}$ & 0.308 & 2.079 & 0.760 & $\mu_{P r}=\mu_{B r}$ \\
3 & $F D E_{T} *$ & $\mu_{P r}=\mu_{B r}$ & $\mu_{P r}>\mu_{B r}$ & 2.989 & 2.570 & 0.030 & $\mu_{P r}>\mu_{B r}$ \\
\hline
\end{tabular}




\section{Results}

Our results showed that predicate tests were the best in terms of fault detecting effectiveness $F D E_{T}$ under normal circumstances where programs have control flow graphs with conditionals. On the other hand they took more test cases i.e. more testing effort $T E_{T}$. Branch tests are more cost effective as the required number of tests is less and giving comparable fault detecting effectiveness to that of predicate tests. As the box-plots for $P I_{T}$ demonstrated, branch tests were quite consistent in terms of their fault detecting capabilities that ensures a level of performance from testing. Block test suites were small in size but were quite inconsistent and have less $F D E_{T}$ than the other two. A useful observation in this experiment is whichever criteria results into minimum number of test cases required, showed highest variability in the performance index. Table 3 shows the three idioms of effectiveness criteria for all five programs obtained as a basis of average taken over all twenty-five test suites for each coverage criteria.

Table 3. Effectiveness Criteria Results

\begin{tabular}{|l|l|l|l|l|l|l|l|l|l|}
\hline Program Name & \multicolumn{3}{|c|}{$F D E_{T}$} & \multicolumn{3}{c|}{$T E_{T}$} & \multicolumn{3}{c|}{$P I_{T}$} \\
\hline & Block & Branch & Pred & Block & Branch & Pred & Block & Branch & Pred \\
\hline \hline HotelManagement & 0.64 & 0.81 & 0.91 & 17.4 & 19.7 & 24.2 & 2.13 & 2.33 & 2.12 \\
PostalCodes & 0.74 & 0.78 & 0.97 & 33.1 & 35.0 & 48.7 & 2.39 & 2.35 & 1.87 \\
CruiseControl & 0.44 & 0.82 & 0.87 & 9.68 & 16.8 & 19.6 & 2.86 & 2.15 & 1.80 \\
JavaVector & 0.92 & 0.99 & 0.84 & 51.7 & 54.5 & 43.0 & 1.33 & 1.32 & 1.35 \\
Monopoly & 0.81 & 0.87 & 0.96 & 29.0 & 31.4 & 33.3 & 1.64 & 1.58 & 1.60 \\
\hline
\end{tabular}

From the bug analysis, we found that bug of the type LCO, VRO, SSO, VRO and AOI are typically revealed by all the three coverage criteria where as LOR and CFD bugs (mainly inserted in conditionals) are poses some challenge to block coverage but likely to be identifiable by branch and predicate coverage. Predicate coverage is especially found to be much more effective in MCO bugs which might be prevalent in conditional statements with multiple conditions.

\section{$5 \quad$ Validity and Generality}

An important issue that greatly affects the effectiveness of writing automated tests is use of proper test oracles. Great care was taken to write automated tests with proper oracles. All the tests were written by first author and since same test pool was used in generating test suites for the coverage criteria under study, we argue that any variability, if present, should not influence our findings. Another factor that can influence the results is the interaction between code under test and test oracles. To control this effect, we did not seed any faults in the code which is used to check test oracles, i.e. JUnit assertions [26].

In terms of external validity, we believe that our results should be general enough to apply at least to other java programs in the context of the type of the 
faults seeded as we have considered considerable number of faults of each type. We have not considered object-oriented specific bugs such as those related with inheritance and dynamic binding as we did not find enough opportunity to seed these faults in the considered programs hence we make no claim regarding these kind of faults. It will be interesting to pursue this issue in another study.

We have considered control structure at a class's method level to obtained information of blocks and branches and hence, method calls were not treated as branches. So our results should also be viewed in this context.

\section{Summary and Conclusions}

The three main control-flow based coverage criteria are - block, branch and predicate coverage. The aim of this work was to study the relative effectiveness of these three criteria. In our experiment, we tested five moderately sized Java programs by generating twenty-five different minimal test suites from a large testpool, for each program-coverage pair. To test a program, we created sufficient number of mutants using a set of applicable mutation operators and tested them with a set of generated minimal coverage test suites for each coverage criteria. The three structural coverage criteria were than compared in terms of their fault detection abilities $\left(F D E_{T}, P I_{T}\right)$ and testing efforts $\left(T E_{T}\right)$ requirements.

We found that Predicate Coverage criterion demonstrated best $F D E_{T}$ but at more cost, i.e. more $T E_{T}$ than the other two. On the other hand, Block coverage criterion took least testing efforts $T E_{T}$, but at lower $F D E_{T}$ than the other two. Branch Coverage criterion performed in between the two in terms of $F D E_{T}$ and $T E_{T}$. Performance Index $P I_{T}$ of block coverage criterion found to be on higher side but with greater variability where as $P I_{T}$ for branch coverage criterion found to be slightly less than that of block criterion but was quite consistent. $P I_{T}$ for predicate test suites was observed to be lower than the other two. Our results show that branch test suites are likely to perform with consistent effectiveness and their effort requirements in terms of test suite size is smaller than that of predicate test suites but larger than the block test suites.

The results obtained from the analysis of $P I_{T}$ for all the three coverage criteria revealed the fact that some block test suites may be more efficient, i.e. revealing faults at par with the other two criteria with smaller test suite size but other may perform worse, thereby being unreliable and in-consistent as compared to the other two criteria. Predicate coverage criterion was found to be least efficient but quite reliable whereas branch criteria demonstrated similar reliability to predicate criteria with better efficiency.

Based on our investigation for choosing a suitable criterion to test a given program, we observed that branch coverage is the best choice for getting better results with moderate testing efforts. The testing effectiveness can be improved considering predicate coverage for the methods containing composite conditionals but with increased efforts requirements.

In this work, we have used a novel approach to randomize the effect of a particular test suite. We generated twenty-five different coverage-adequate test 
suites from a test-pool for each coverage criterion. The test suites were algorithmically constructed in a manner that they contained minimum number of test cases, thereby, enabling us to do a uniform analysis. This also facilitates computation of performance indexes for the coverage test suites.

Based on our experience of testing of a well designed object-oriented program like Monopoly, we argue that such programs are more robust as we found relatively few places where we can insert faults related to the mutation operators considered in this study. Also because of small methods implementing single operation, the three testing approaches demonstrated similar effectiveness. It will be interesting to investigate the effectiveness of these coverage criteria and other testing approaches to test object-oriented programs with object-orientedspecific bugs like related with hierarchies, dynamic binding, method over-riding, etc. Also similar studies to be replicated to demonstrate the applicability of our results

\section{Acknowledgement}

The authors would like to thank L. Raghu, for providing enough help to use his tool JavaCoverage that he developed as part of his M Tech thesis at IIT Kanpur.

\section{References}

1. Andrews J.H., Briand L. C. and Y. Labiche Is mutation an appropriate tool for testing experiments? In Proc. Int'l.Conf. Softw. Eng ICSE pages 402-411, May 2005.

2. Antoniol G at al A Case Study Using the Round-Trip Stretegy for State-Based Class Testing In Proc of the 13th Int'l Symp. On Reliability ISSRE'02.

3. Briand L.C., Labiche Y. and Wang Y. Using Simulation to Empirically Investigate Test Coverage Criteria Based on Statechart In Proc of the 26th Int'l Conf. on Software Engineering ICSE 2004.

4. Barbara A. Kitchham, Shari Lawrence Pfleeger, Lesley M. Pickard, Peter W. Jones, David C. Hoaglin, Khaled El Emam, Jarrett Rosenberg Preliminary Guidelines for Empirical Research in Software Engineering In IEEE Trans. on Software Engineeing Vol.28, No.8, August 2002.

5. Basili V.R.,R.W. Silby, David H. Huchens Experimentation in Software Engineering In IEEE Trans. on Software Engineeing Vol.SE-12, No.7, July 1986.

6. Basili V.R. and Selby R.W. Comparing the effectiveness of software testing In IEEE Transactions on Software Engineering Vol. SE-13, No.12, pages 1278-1296, December 1987.

7. Douglas C. Montgomery Design and Analysis of Experiments fifth edition, John Wiley and Sons Inc., 2001.

8. Fowler M. Refactoring: Improving the Design of Existing Code Addison Wesley, 1999.

9. Frankl P.G. and Weiss S.N. An experimental comparison of the effectiveness of branch testing and data flow testing In IEEE Transactions on Software Engineering Vol. 19, No.8, pages 774-787, August 1993. 
10. Frankl P.G. and Weyuker J.E. An applicable family of data flow testing criteria In IEEE Transactions on Software Engineering Vol. SE-14, No.10, pages 1483-1498, October 1988.

11. Frankl P.G. and Weyuker J.E. A formal analysis of the fault-detecting ability of testing methods In IEEE Transactions on Software Engineering Vol 19, No.3, pages 202-213, March 1993.

12. Frankl P.G. and Weyuker J.E. Provable improvements on branch testing In IEEE Transactions on Software Engineering Vol 19, No.10, pages 962 - 975, 1993.

13. Goodenough J.B. and Garhart S.L. Toward a theory of test data selection In IEEE Transactions on Software Engineering Vol SE-3, 1975.

14. Hamlet R. Theoretical comparison of testing methods In Proceedings of SIGSOFT Symposium on Software Testing, Analysis and Verification pages 28-37, December 1989.

15. Hong Zhu, Patrick A.V. Hall, John H.R. May. Software Unit Test Coverage and Adequacy In ACM Computing Surveys Vol.29, No.4, December 1997.

16. Kim S, Clark J.A. and McDermid J.A. The Rogorous Generation of Java Mutation Using HAZOP In Proc. ICSSEA- 3 pages 9-10(11), 1999.

17. M. Hutchins, H. Foster, T. Goradia, and T. Ostrand. Experiments on the effectiveness of dataflow- and controlflow-based test adequacy criteria. In Proceedings of the 16th International Conference on Software Engineering ICSE May 1994.

18. Natalia Juristo, Ana M. Moreno and Sira Vegas Reviewing 25 Years of Software Testing Experiments In Jour. Empirical Software Engineering Vol 9, 1-2 pp 7-44 March 2004.

19. Ntafos, S.C. An evaluation of required element testing strategies In Proceedings of the Seventh International Conference on Software Engineering Pages 250-256.

20. Pankaj Jalote An Integrated Approach to Software Engineering second edition, Narosa Publishing House, 1999.

21. Paul D. Berger, R.E. Maurer Experimental Design Thomson Duxbury, 2002.

22. Raghu L. Testing Changes made to the Code using Coverage Data, M Tech Thesis, Dept of CSE, IIT Kanpur June 2005.

23. Weyuker E.J. Can We Measure Software Testing Effectiveness? In Proceedings of IEEE-CS International Software Metrics Symposium pages 100-107, May 1993.

24. Weyuker E.J., Weiss S.N., Hamlet D. Comparison of Program Testing Strategies In Proceedings of the Fourth Symposium on Software Testing, Analysis and Verification Pages 154-164, October 1991.

25. Wood M., Mark Roper, Andrew Brooks, James Miller Comparing and combining software defect detection techniques: A Replicated Experimental Study In ACM SIGSOFT Software Engineering Notes Vol. 22, No. 6, November 1997.

26. JUnit Home Page http://www.junit.org

27. http://www-dse.doc.ic.ac.uk/concurrency/book- applets/CruiseControl.html

28. http://open.ncsu.edu/se/monopoly/ 


\section{Appendix}
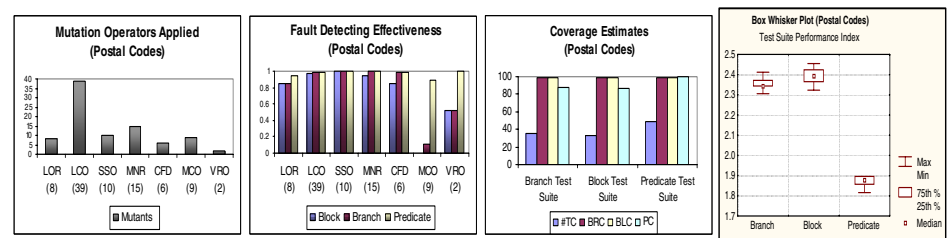

(a) Graphical Analysis for PostalCode data
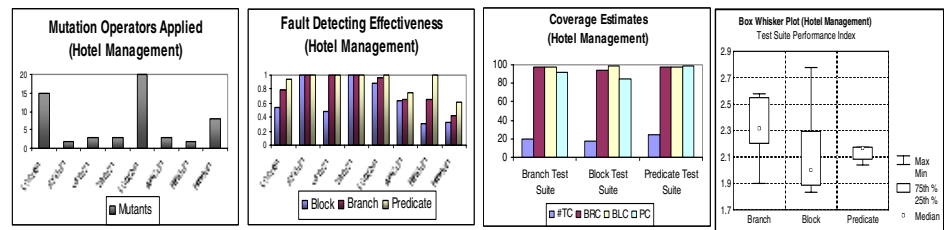

(b) Graphical Analysis for HotelManagement data
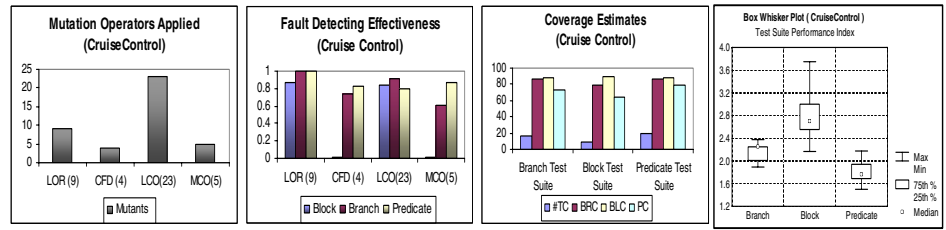

(c) Graphical Analysis for CruiseControl data
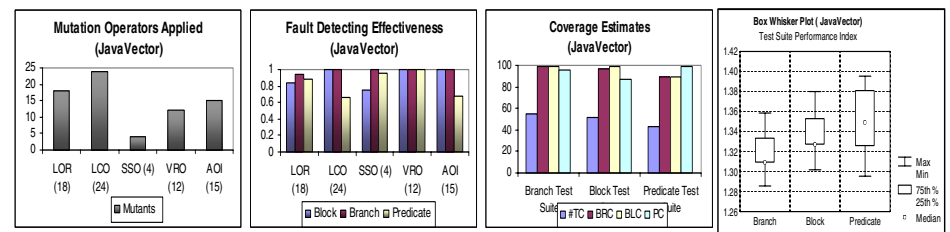

(d) Graphical Analysis for JavaVector data
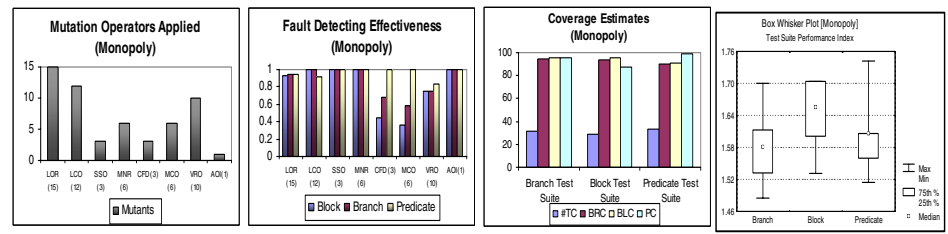

(e) Graphical Analysis for Monopoly data

Fig. 1. Program-wise Graphical Analysis of Experimental Data (left-to-right) (i) Faults applied (ii) $\mathrm{FDE}_{\mathrm{T}}$ of Coverage Test Suites (iii) All-Coverage information of Coverage Test Suites (iv) Performance Index $\mathrm{PI}_{\mathrm{T}}$ 